\title{
SEX-BIASED DISPERSAL IN A MIGRATORY BAT: A CHARACTERIZATION USING SEX-SPECIFIC DEMOGRAPHIC PARAMETERS
}

\author{
Eric Petit, ${ }^{1,2}$ Francois Balloux, ${ }^{3}$ and Jerome Goudet ${ }^{1}$ \\ ${ }^{1}$ Institute of Ecology (Zoology and Animal Ecology), Biology Building, University of Lausanne, \\ 1015 Lausanne, Switzerland \\ ${ }^{3}$ Institute of Zoology, University of Bern, 3032 Hinterkappelen-Bern, Switzerland
}

\begin{abstract}
We studied the noctule bat (Nyctalus noctula), in which the mitochondrial $F_{\mathrm{ST}}$ is about 10 times that revealed by nuclear markers, to address two questions. We first verified whether random dispersal of one sex is compatible with highly contrasted mitochondrial and nuclear population structures. Using computer simulations, we then assessed the power of multilocus population differentiation tests when the expected population structure departs only slightly from panmixia. Using an island model with sex-specific demographic parameters, we found that random male dispersal is consistent with the population structure observed in the noctule. However, other parameter combinations are also compatible with the data. We computed the minimum sex bias in dispersal (at least $69 \%$ of the dispersing individuals are males), a result that would not be available if we had used more classical population genetic models. The power of multilocus population differentiation tests was unexpectedly high, the tests being significant in almost $100 \%$ of the replicates, although the observed population structure infered from nuclear markers was extremely low $\left(F_{\mathrm{ST}}=0.6 \%\right)$.
\end{abstract}

Key words.-Chiroptera, dispersal, F-statistics, Nyctalus noctula, population structure, power analysis, test of population differentiation.

Received May 2, 2000. Accepted October 27, 2000.

Owing to their high variability (Jarne and Lagoda 1996) and to the parallel development of statistical methodologies whose power increases constantly (Rousset and Raymond 1997; Luikart and England 1999), microsatellites provide population geneticists with very powerful tools to detect population subdivision. This may be of great help in detecting genetic structure in species characterized by high vagility such as birds or migratory bats, in which typical estimates of population subdivision as assessed with nuclear genetic markers $\left(F_{S T}^{n u c}\right)$ average around 1.6-2.2\% (Barrowclough 1983; Webb and Tidemann 1996; Petit and Mayer 1999). However, low but statistically significant population structures should be interpreted cautiously because it is questionable whether such results correspond to any biologically meaningful phenomenon (large Type I errors; Hedrick 1999). Nonetheless, if the detected phenomenon has a biological basis, the more powerful statistical tests are, the more accurately population structures can be described.

Nyctalus noctula provides one example of a species with an extremely low population structure. In spring and summer, noctule bats form nurseries (which we will consider as populations throughout this paper) that consist of females with their offspring. In late summer and autumn, these individuals migrate to hibernacula that are located further south, flying along migration routes whose direction is quite constant across Europe (Petit and Mayer 2000). During this journey, migrating females mate with males that have dispersed from their natal nursery, providing an opportunity for gene flow to occur among nurseries. Over its European distribution range, this migratory bat species is characterized by a nuclear $F_{\mathrm{ST}}$ (estimated with microsatellites) of only $0.6 \%$, but none-

\footnotetext{
2 Present address: Ethology-Evolution-Ecology, UMR 6552, University of Rennes I, F-35042 Rennes, France; E-mail: eric.petit@ univ-rennes1.fr.
}

theless significantly different from zero (Petit and Mayer 1999). Based on a comparison of these data with an estimate of mitochondrial structure, Petit and Mayer (1999) suggest that male noctules disperse randomly across Europe. Is random dispersal of one sex consistent with a significant population structure detected with nuclear markers? Hedrick (1999) points out how easy it is to overinterpret significant results when the statistical power to detect a known biological effect has not been assessed (risk of Type I errors). Therefore, to answer the above question, we assessed the statistical power of population differentiation tests using simulated noctule populations.

To this end, we need to obtain estimates of population size and dipersal rate in the noctule. In this species, the discrepancy between mitochondrial and nuclear genetic structures indicates that dispersal is male biased (Petit and Mayer 1999). The effective sex ratio (the ratio of the effective number of males to the effective number of females) is also likely to be biased. During the mating season, male noctules defend a territory against other males and advertise loudly using specific mating calls to attract females to their roost (Sluiter and van Heerdt 1966; Zingg 1988; Gebhard 1997, pp. 245260). This among-male competition suggests that there should be a large variance in male mating success. The occurrence of nonterritorial adult male groups during the mating season supports this view (Gebhard 1997, pp. 251-252). In contrast, almost all adult females reproduce each year (Heise 1989). Thus, because the sex ratio at birth is balanced (Heise 1989 ) and we assume equal survival rates for males and females, the effective sex ratio is likely to be female biased. Although it has long been recognized that sex-biased dispersal is a common feature of most mammal and bird species (Greenwood 1980) and that random mating is not widespread, only recently have population genetic models included sexspecific parameters that can account for these asymmetries 
(Chesser 1991a,b; Chesser et al. 1993; Chesser and Baker 1996; Wang 1997). The noctule is a typical mammal species, in which equal female and male dispersal and balanced effective sex ratio are unlikely. Thus, rather than a classic island model, we used an island model that accounts for sex differences to define the parameters that were further used to simulate noctule populations.

In this paper, we proceeded as follows. First the available mitochondrial and nuclear data allowed us to estimate the effective numbers of dispersing males and females according to the island model with sex-specific demographic parameters (Wang 1997; Petit and Mayer 1999; Petit et al. 1999). Then, noctule bat populations were simulated using the estimated demographic parameters. Finally, population structure analyses were conducted on the simulated data as for the real dataset to estimate the power of the analysis we conducted on the noctule dataset.

\section{Materials AND Methods}

\section{Estimation of Sex-Specific Demographic Parameters}

Female dispersal rate, $d_{f}$, was estimated from the mitochondrial DNA data of seven central and eastern European populations (Petit et al. 1999). The population differentiation parameter $F_{\mathrm{ST}}^{m t}$ was estimated from Weir and Cockerham's (1984) $\theta$ using FSTAT (vers. 2.8 , Goudet 1995). $d_{f}$ was obtained by solving the following quadratic equation, which stems from the equilibrium value for $F_{\mathrm{ST}}^{m t}$ in an island model (Hartl and Clark 1997, eq. 5.16):

$$
\begin{aligned}
& {\left[\left(N_{f}-1\right) F_{\mathrm{ST}}^{m t}+1\right] d_{f}^{2}-2 d_{f}\left[\left(N_{f}-1\right) F_{\mathrm{ST}}^{m t}+1\right]+\left(1-F_{\mathrm{ST}}^{m t}\right)} \\
& \quad=0
\end{aligned}
$$

where $N_{f}$ is the effective number of females per population.

Wang (1997) derived a model of population structure that allows for different numbers of females and males and for different rates of dispersal by each sex. For an island model of dispersal at equilibrium and assuming relatively large number of males and females, Wang (1997) obtained the following expectation for $F_{\mathrm{ST}}^{\text {nuc }}$ :

$$
\begin{aligned}
& \frac{1}{F_{\mathrm{ST}}^{n u c}}=\left(d_{m}+d_{f}\right)\left(4-d_{m}-d_{f}\right) \\
& \times\left(\frac{2 N_{m} N_{f}}{N_{m}+N_{f}}\right. \\
& \quad+\left\{\left[N_{f}\left(1-d_{m}\right)\left(1-2 d_{m}+d_{f}\right)\right.\right. \\
& \left.\quad+N_{m}\left(1-d_{f}\right)\left(1-2 d_{f}+d_{m}\right)\right] \\
& \left.\left.\quad \div\left[\left(N_{m}+N_{f}\right)\left(d_{m}+d_{f}\right)\left(4-d_{m}-d_{f}\right)\right]\right\}\right)-\frac{1}{s},
\end{aligned}
$$

where $N_{f}$ and $N_{m}$ are the effective numbers of females and males, $d_{f}$ and $d_{m}$ are the effective female and male dispersal rates, and $s$ is the number of populations.

For an estimate of $F_{S T}^{n u c}$, we used the result of Petit and Mayer (1999), whereas we used the results of Petit et al. (1999) for $F_{\mathrm{ST}}^{m \text {. }}$ Clearly, with equations (1) and (2) it is im- possible to obtain a unique solution for five variables. We therefore used our knowledge of the species to adjust some of them (see below). In all cases, the number of subpopulations, $s$, has been fixed to 100 . This is quite arbitrary but it turns out that Wang's equation is rather insensitive to the number of populations if it is larger than 50 (see eq. 2), which is certainly the case in the noctule bat. For the remaining four variables, we assumed first that the sex ratio was balanced $\left(N_{f}=N_{m}\right)$. We sought solutions for the remaining variables $N_{f}=N_{m}$ and $d_{f}$ when male dispersal is maximum $\left(d_{m}=1\right)$. This sets the lower limit for $N_{f}=N_{m}$. To solve the equations when the only constraint on sex ratio was that there should be more females than males (the reverse makes no sense biologically; Heise 1989), we fixed $N_{f}$ to 300 , the average size of nurseries (E. Petit and F. Mayer, unpubl. data). Note that the effective number of females is likely to be close to their census number because almost all females reproduce each year (Gaisler et al. 1979; Heise 1989). We were left with one equation and two unknowns, $N_{m}$ and $d_{m}$. Pairs of solutions were obtained under the constraint that $N_{m}$ should be smaller than $N_{f}$. We also checked whether our results were sensitive to changes in $N_{f}$. Finally, confidence intervals were computed by taking the upper and lower $95 \%$ bootstrap confidence limits of $F_{\text {ST }}^{\text {nuc }}$ ( 0.013 and 0.001 , respectively) to solve Wang's equation.

\section{Simulations}

\section{Simulation parameters}

All simulations were performed with the shareware EASYPOP (Balloux 1999). This individual-based model allows us to simulate multilocus population datasets under a large array of conditions. In particular, it allows us to specify the number of males and females per population, the sex-specific amount of dispersal, and the mode of reproduction (polygyny, monogamy, promiscuity). We used EASYPOP to perform simulations under three distinct scenarios (see below for a justification): (1) 1:1 effective sex ratio with 51 males and 51 females, and random male dispersal; (2) female-biased sex ratio with 300 females and 30 males, and random male dispersal; and (3) 1:1 sex ratio with 300 males and 300 females, and limited male dispersal. Female dispersal was estimated with equation (1) from mitochondrial data. In addition to the test simulations described above, we also ran control simulations in which dispersal was random for both sexes. Within-population mating was modeled as random because $F_{\text {IS }}$ estimates were close to zero (Petit and Mayer 1999). Dispersal occurred according to an island model because isolation by distance could be detected neither in the nuclear (Petit and Mayer 1999) nor in the mitochondrial dataset (data not shown). The number of populations was fixed to 100 . Each individual was characterized by eight unlinked loci, as in the real dataset, mutating according to a $\mathrm{K}$ Allele Model (KAM, Estoup and Cornuet 1999). The mutation rate was set to $10^{-3}$ (Jarne and Lagoda 1996), and the number of possible allelic states was restricted to 30 (in the real dataset, the number of alleles per locus ranged from 10 to 25). As long as the mutation rate is orders of magnitude lower than the dispersal rate, which is the case here, the model chosen to generate new mutations is not expected to substantially in- 
TABLE 1. Results of the population structure analysis of the simulated datasets. For each parameter combination, 120 replicates were run. Balanced sampling corresponded to 20 females sampled in each of 13 populations, and unbalanced sampling was a combination of four populations with 20 females each, four populations with 14 females each, and five populations with eight females each. $\theta$ and $G$ were estimated and tested using FSTAT (vers. 2.8, Goudet 1995), and the result given here for $\theta$ is the mean over the 120 replicates (standard deviations were equal to 0.001, apart for the test situations with unbalanced sampling marked with an asterisk, for which standard deviations were equal to 0.002). In the actual dataset, $\theta$ was equal to 0.006 and highly significant (Petit and Mayer 1999), as was the $G$-statistic.

\begin{tabular}{|c|c|c|c|c|c|c|c|}
\hline \multirow[b]{2}{*}{$\begin{array}{l}\text { No. females/ } \\
\text { No. males }\end{array}$} & \multirow[b]{2}{*}{$d_{f} / d_{m}$} & \multicolumn{3}{|c|}{ Balanced sampling } & \multicolumn{3}{|c|}{ Unbalanced sampling } \\
\hline & & $\theta$ & $\begin{array}{c}P(\theta) \\
<0.05(\%)\end{array}$ & $\begin{array}{c}P(G) \\
<0.05(\%)\end{array}$ & $\theta$ & $\begin{array}{c}P(\theta) \\
<0.05(\%)\end{array}$ & $\begin{array}{c}P(G) \\
<0.05(\%)\end{array}$ \\
\hline \multicolumn{8}{|l|}{ Test } \\
\hline \multicolumn{8}{|l|}{ Control } \\
\hline
\end{tabular}

fluence population differentiation estimators. For each condition, 120 replicates were computed. The genotypes were analyzed after 1500 generations, when all the relevant statistics had reached equilibrium. This is very likely the case for noctule populations because they recolonized Europe at least 3000 years ago (i.e., about 3000 generations; Petit et al. 1999).

\section{Data analysis}

For each replicate, we conducted the same type of analysis as for the real data (Petit and Mayer 1999) using a slightly modified version of FSTAT (vers. 2.8, Goudet 1995) that can handle multiple datasets. Twenty females from each of 13 populations were sampled at random in each simulation run. We computed $\theta$ (Weir and Cockerham's [1984] estimator of $\left.F_{\mathrm{ST}}\right)$ and tested whether it departed from zero. In most empirical studies, sampling is not as balanced as the ideal sampling scheme we used to analyze the simulations. Therefore, we also carried out a multilocus version of the $G$-test described in Goudet et al. (1996) because it has been shown to be more powerful than tests based on $F_{\mathrm{ST}}$ when sampling is unbalanced, and we assessed the power of both tests with an unbalanced sampling scheme (see Table 1 for details). The

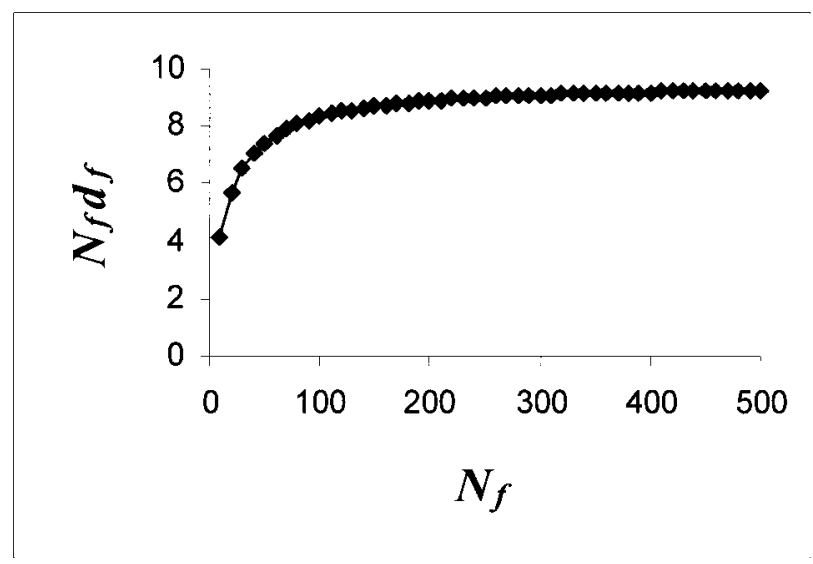

FIG. 1. Effective number of dispersing females. This curve gives the solutions to equation (1). multilocus $G$-statistic is simply the sum over loci of the individual locus $G$-statistics:

$$
G=-2 \sum_{k-1}^{n p} \sum_{i-1}^{n u} n_{i k} \ln \left(\frac{n_{i k}}{n_{k} p_{i}}\right),
$$

where $n_{i k}$ is the number of occurences of allele $i$ in sample $k, n_{k}$ is the number of alleles (twice the number of individuals) in sample $k$, and $p_{i}$ is the frequency of allele $i$ in the whole dataset. Significance testing was performed by permuting multilocus genotypes among samples (10,000 permutations). A multilocus $G$-statistic was calculated for each permuted dataset, and the proportion of permuted statistics larger or equal to the observed gave an estimate of the sought probability.

\section{RESULTS}

\section{Estimation of Sex-Specific Demographic Parameters}

The estimate of $F_{\mathrm{ST}}^{m t}$ was significantly different from zero and had a value of 0.05 (jackknifing over populations gave a standard error of 0.008) for the seven eastern and central European populations. Solving equation (1) yielded an effective number of dispersing females of 4.1, 9.1, and 9.5 for $N_{f}=10, N_{f}=300$, and $N_{f} \rightarrow \infty$ respectively (Fig. 1).

The set of demographic parameters that satisfy Wang's (1997) equation are shown in Figure 2. We first looked for a solution constrained by $N_{m}=N_{f}$ and random male dispersal $\left(d_{m}=1\right)$. This solution corresponds to the uppermost point of Figure 2 (diamond), where populations consist of 51 individuals of each sex. Note that this point sets the upper bound for $N_{m} d_{m}$ at 51 dispersing males per generation. It also provides the maximum proportion of dispersing males (= $N_{m} d_{m} /\left[N_{m} d_{m}+N_{f} d_{f}\right]$ ), which is $87 \%$. Additional solutions were obtained by setting $N_{f}=300$, a biologically meaningful value for $N$. noctula, with the constraints of female-biased sex ratio and $d_{m} \leq 1$. This latter constraint sets the lowest effective sex ratio at which a solution can be found, because $d_{m}$ increases when $N_{m} / N_{f}$ decreases. For populations of 300 females, a solution to equation (2) is obtained for all sex ratios, meaning that any number of males can lead to an $F_{\text {ST }}^{\text {nuc }}$ of $0.6 \%$. However, the number of effective migrant males 


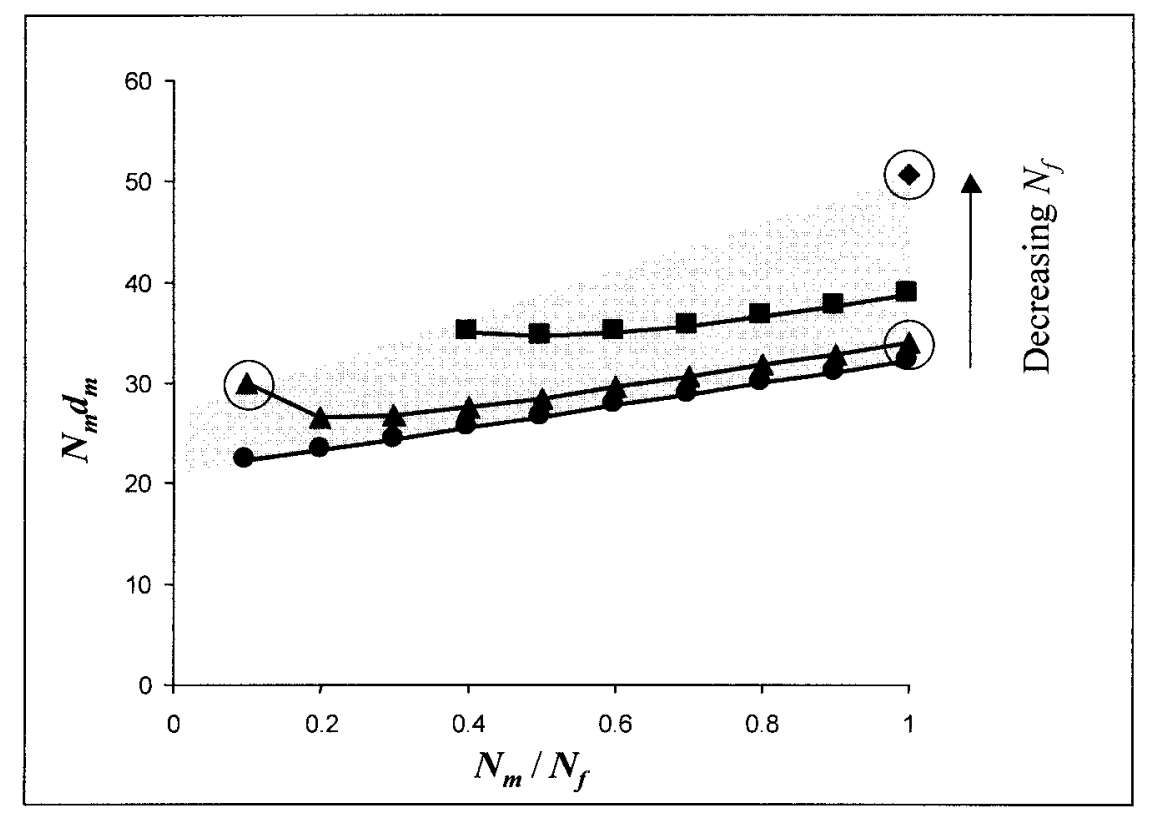

FIG. 2. Solutions to Wang's (1997) equation for the noctule bat. The diamond represents $N_{f}=51$, squares represent $N_{f}=100$, triangles represent $N_{f}=300$, and dots represent $N_{f}=3000$. To solve Wang's equation, the number of populations $(s)$ was set to 100 and the female dispersal rate $\left(d_{f}\right)$ was obtained from equation (1). The three situations that were simulated with EASYPOP (Balloux 1999) are indicated by circles, and the area occupied by the set of solutions to Wang's equation is shown in gray.

remains remarkably constant across this range of sex ratio values; it varies between 26 for a sex ratio of $0.2\left(N_{m}=60\right)$ to 34 for a balanced sex ratio $\left(N_{m}=300\right)$. Finally, we investigated the effect of $N_{f}$. It can be seen from Figure 2 that as $N_{f}$ decreases, male dispersal increases, and the possible sex ratios become restricted. In contrast, increasing $N_{f}$ above 300 has only minor effects. When $N_{f} \rightarrow \infty, N_{m} d_{m}$ cannot go below 21 . Note that this situation gives the minimum proportion of dispersing males, which is $69 \%$. Using the $95 \%$ confidence interval of $F_{\mathrm{ST}}^{n u c}$ led to the following confidence limits for $N_{m} d_{m}$ : the confidence interval for the lower boundary, 21, was 9-125 and the confidence interval for the upper boundary, 51, was 22-326.

Overall, the solutions to Wang's (1997) equation roughly define a triangle in the parameter space (gray area on Fig. 2). It is limited on its right by the constraint that $N_{m} \leq N_{f}$, above by the constraint that $d_{m} \leq 1$, and underneath by the fact that it is a region with no solution. Three sets of parameters (indicated by circles on Fig. 2) were chosen in this parameter space for the simulations (Table 1), which correspond roughly to the three angles of the triangle. This allowed us to assess the power of tests of population differentiation when $F_{\mathrm{ST}}^{n u c}$ is around $0.6 \%$, and to check whether these tests are equally powerful across the space of meaningful parameter combinations.

\section{Simulations}

The results of the simulations (Table 1) show that for all three parameter combinations significant population structure (as assessed by a nominal level of 5\%) was detected in $100 \%$ of the cases when sampling was balanced. Unbalanced sampling slightly reduced the power of the tests, more substantially so when $N_{m}=N_{f}=51$. In this case, the average $\theta$ is
1.5 times lower than in the other scenarios, which might explain the difference in power. As for the single-locus situation (Goudet et al. 1996), the multilocus $G$-test outperforms the test based on $\theta$ when sampling is unbalanced. In addition to these test simulations, control simulations corresponding to random dispersal of both sexes were performed. As expected in such cases, mean $\theta$ was zero and around 5\% (range: $2.5-8.3 \%$ ) of the tests were significant at the $5 \%$ nominal level.

\section{DISCUSSION}

Our study first confirms that tests of genetic structure are very powerful, allowing us to detect differentiation even when 50 individuals of one sex and 10 individuals of the other sex disperse per generation. Our power analysis further indicates that, if sampling is unbalanced, it is better to use the $G$-test than the test based on $\theta$. However, if our results are qualitatively similar to that found in the single-locus situation (Goudet et al. 1996), the multilocus tests are more powerful than the single-locus tests and the difference between both tests is conspicuous only for lower values of $F_{\text {ST }}$ in the multilocus case. Finally, these analyses show that random male dispersal is indeed consistent with the genetic structure observed in the noctule bat. Nevertheless, other parameter combinations are compatible with the data. For instance, for $N_{f}=300$, both a female-biased sex ratio with random male dispersal and a balanced sex ratio with low male dispersal are consistant with the observed genetic structure.

Thus, independent information either on male dispersal rates or on the effective sex ratio would allow to situate the noctule more precisely in the space of possible parameter combinations. In migratory bats, the roosts that are situated 
on migration routes are a resource that is of paramount importance to females. If males mate with females that do not survive migration, their fitness is zero. Thus, one could easily imagine that the mating system has evolved to provide females with a greater chance to survive migration. If this were true, males should be selected to offer the best possible roosts to migrating females. This is consistent with the fact that male noctules defend a territory and attract females to this territory while the females are migrating (Sluiter and van Heerdt 1966; Gebhard 1997). Mating roosts are in no way (neither behaviorally nor geographically) related to nurseries. Therefore, natal philopatry should not evolve in male noctules. Furthermore, competition among males is likely to lead to biased effective sex ratios in the noctule. Altogether, this suggests that noctule populations are expected to have high male dispersal rates and female-biased sex ratios. Clearly, however, further quantitative and independent evidence is needed to situate with some confidence the noctule on the continuum defined by Wang's (1997) model.

A parameter that is limited to quite a narrow range by Wang's (1997) equation is the effective number of dispersing males, which is restricted to values between 21 and 51. However, these quantitative estimates should be taken cautiously. Taking the upper and lower $95 \%$ bootstrap confidence limits of $F_{\text {ST }}^{n u c}$ to solve Wang's equation resulted in very large confidence intervals for these boundaries. When $F_{\mathrm{ST}}$ is close to zero, even small absolute confidence intervals for $F_{\text {ST }}$ convert into very large confidence intervals for demographic parameters (Whitlock and McCauley 1999). This stresses the importance of field observations if one wishes to make good predictions on the mean number of individuals that live in or disperse between populations that are very slightly differentiated.

Beside the problem of large confidence intervals, an additional difficulty comes from the particular population structure encountered in the noctule and other bat species. The genetic structure of hibernacula gives evidence for the mixing of females during fall migration. Whereas 5.3\% $(P<0.0001)$ of the mitochondrial genetic variance exists among central and eastern European nurseries, no population structure can be detected among hibernacula containing individuals that come from these nurseries (among-hibernacula variance is $0.9 \%$ and not significantly different from zero; Petit and Mayer 2000). Therefore, a particular male is more likely to mate with 10 females coming from 10 different nurseries than with 10 females all coming from the same colony. This differs from existing models with or without social structure because they implicitly assume that individuals belong to a defined population. In the noctule bat, however, males are genetically so similar to each other (recall that $F_{\mathrm{ST}}$ is significant but very low) that there is hardly any difference between the two models. It is noteworthy that this conclusion may not hold for species with similar population structure but stronger population differentiation. In species in which males cannot be assigned to a defined population and population differentiation is substantial, specific models need to be developed.

Models that take into account the complexity of social systems and the differences between males and females allow information that is hidden in more classical models such as Wright's (1931) island model to be uncovered. In the black- tailed prairie dog (Cynomys ludovicianus), it has been shown using Chesser's models (Chesser 1991a,b; Chesser et al. 1993) that male dispersal associated with polygyny leads to the formation of high coancestry levels with negligible inbreeding (Sugg et al. 1996; Dobson et al. 1997). Such a situation makes the evolution of sociality possible without having to pay the costs of inbreeding depression. Balloux et al. (1998) used a similar approach to show that in the monogamous and female-dispersed greater white-toothed shrew (Crocidura russula) sex-biased dispersal strongly influences the effective size of populations and their ability to retain genetic variance. Using Wang's (1997) model, we have quantified the sex bias in dispersal for the noctule, showing that the population structure observed in this species corresponds to a minimum proportion of dispersing males of $69 \%$. If we had simply converted the observed nuclear $F_{\mathrm{ST}}$ into an estimate of dispersal, we would have concluded that, on average, 41.4 individuals disperse from noctule bat populations each generation (in the classical island model, $F_{\mathrm{ST}}=1 /[4 \mathrm{Nm}$ +1 ]; Wright 1931). Knowing that dispersal is sex-biased in most bird and mammal species (Greenwood 1980), this estimate would have not been of much help for understanding dispersal in the noctule bat. These three examples highlight that the social-structure approach of population genetics (Balloux et al. 1998) is very promising. Although rarely used, it generates nontrivial results that are relevant to our understanding of the evolution of dispersal rates and social systems, and as such should be more widely considered.

\section{ACKNOWLEDGMENTS}

We thank T. Burland, G. Heckel, D. Hosken, F. Mayer, and N. Perrin for their valuable comments on earlier drafts of this paper. JG and EP are financially supported by the Swiss National Science Foundation (grant 31-55945.98 to JG and 31-055475.98 to EP).

\section{Literature Cited}

Balloux, F. 1999. EASYPOP: a software for population genetics simulation. Institute of Ecology, University of Lausanne, Switzerland.

Balloux, F., J. Goudet, and N. Perrin. 1998. Breeding system and genetic variance in the monogamous, semi-social shrew, Crocidura russula. Evolution 52(4):1230-1235.

Barrowclough, G. F. 1983. Biochemical studies of microevolutionary processes. Pp. 223-261 in A. H. Brush and G. A. Clark Jr., eds. Perspectives in ornithology. Cambridge Univ. Press, New York.

Chesser, R. K. 1991a. Gene diversity and female philopatry. Genetics 127:437-447.

- $1991 \mathrm{~b}$. Influence of gene flow and breeding tactics on gene diversity within populations. Genetics 129:573-583.

Chesser, R. K., and R. J. Baker. 1996. Effective sizes and dynamics of uniparentally and diparentally inherited genes. Genetics 144: $1225-1235$.

Chesser, R. K., O. E. Rhodes, D. W. Sugg, and A. Schnabel. 1993. Effective sizes for subdivided populations. Genetics 135: 1221-1232.

Dobson, F. S., R. K. Chesser, J. L. Hoogland, D. W. Sugg, and D. W. Foltz. 1997. Do black-tailed prairie dogs minimize inbreeding? Evolution 51:970-978.

Estoup, A., and J.-M. Cornuet. 1999. Microsatellite evolution: inferences from population data. Pp. 49-65 in D. B. Goldstein and C. Schlötterer, eds. Microsatellites: evolution and applications. Oxford Univ. Press, Oxford, U.K. 
Gaisler, J., V. Hanák, and J. Dungel. 1979. A contribution to the population ecology of Nyctalus noctula (Mammalia: Chiroptera). Acta Sc. Nat. Brno 13:1-38.

Gebhard, J. 1997. Fledermäuse. Birkhäuser, Basel.

Goudet, J. 1995. FSTAT (Version 1. 2): a computer program to calculate $F$-statistics. J. Hered. 86:485-486.

Goudet, J., M. Raymond, T. de Meeus, and F. Rousset. 1996. Testing differentiation in diploid populations. Genetics 144: 1933-1940.

Greenwood, P. J. 1980. Mating systems, philopatry and dispersal in birds and mammals. Anim. Behav. 28:1140-1162.

Hartl, D. L., and A. G. Clark. 1997. Principles of population genetics. 3rd ed. Sinauer Associates, Sunderland, MA.

Hedrick, P. W. 1999. Highly variable loci and their interpretation in evolution and conservation. Evolution 53:313-318.

Heise, G. 1989. Ergebnisse reproduktionsbiologisher Untersuchungen am Abendsegler (Nyctalus noctula) in der Umgebung von Prenzlau/Uckermark. Nyctalus 3:17-32.

Jarne, P., and P. J. L. Lagoda. 1996. Microsatellites, from molecules to populations and back. Trends Ecol. Evol. 11:424-429.

Luikart, G., and P. R. England. 1999. Statistical analysis of microsatellite DNA data. Trends Ecol. Evol. 14:253-255.

Petit, E., and F. Mayer. 1999. Male dispersal in the noctule bat (Nyctalus noctula): where are the limits? Proc. R. Soc. Lond. B 266:1717-1722.

2000. A population genetic analysis of migration: the case of the noctule bat (Nyctalus noctula). Mol. Ecol. 9:683-690.
Petit, E., L. Excoffier, and F. Mayer. 1999. No evidence of bottleneck in the postglacial recolonization of Europe by the noctule bat (Nyctalus noctula). Evolution 53:1247-1258.

Rousset, F., and M. Raymond. 1997. Statistical analyses of population genetic data: new tools, old concepts. Trends Ecol. Evol. $12: 313-317$.

Sluiter, J. W., and P. F. van Heerdt. 1966. Seasonal habits of the noctule bat (Nyctalus noctula). Arch. Néerl. Zool. 16:423-439.

Sugg, D. W., R. K. Chesser, F. S. Dobson, and J. L. Hoogland. 1996. Population genetics meets behavioral ecology. Trends Ecol. Evol. 11:338-342.

Wang, J. 1997. Effective size and $F$-statistics of subdivided populations. II. Dioecious species. Genetics 146:1465-1474.

Webb, N. J., and C. R. Tidemann. 1996. Mobility of Australian flying-foxes, Pteropus spp. (Megachiroptera): evidence from genetic variation. Proc. R. Soc. Lond. B 263:497-502.

Weir, B. S., and C. C. Cockerham. 1984. Estimating $F$-statistics for the analysis of population structure. Evolution 38:1358-1370.

Whitlock, M. C., and D. E. McCauley. 1999. Indirect measures of gene flow and migration: $F_{\mathrm{ST}} \neq 1 /(4 N m+1)$. Heredity 82 : $117-125$.

Wright, S. 1931. Evolution in Mendelian populations. Genetics 16: 97-159.

Zingg, P. E. 1988. Eine auffällige Lautäusserung des Abendseglers, Nyctalus noctula (Schreber) zur Paarungszeit (Mammalia: Chiroptera). Revue suisse Zool. 95(4):1057-1062.

Corresponding Editor: M. Douglas 